\title{
Cost-Delay Tradeoffs for Two-Way Relay Networks
}

\author{
Ertugrul Necdet Ciftcioglu, Student Member, IEEE, Yalin Evren Sagduyu, Member, IEEE, \\ Randall A. Berry, Member, IEEE, and Aylin Yener, Member, IEEE
}

\begin{abstract}
We consider two sources in a wireless network exchanging stochastically varying traffic using an intermediate relay. Each relay use incurs some cost, which, for example, could be transmission energy. This cost is shared between the sources when packets from both are transmitted simultaneously by the relay using network coding. If the relay transmits a packet originating from one source only, the cost is incurred by that source only. In this setting, we study transmission policies that tradeoff the average cost with the average packet delay. We first present the cost-delay tradeoff for a centralized scheme using Lyapunov stability arguments. Next, we consider a distributed policy, where each source aims to optimize its own cost-delay tradeoff. We determine the Nash equilibrium of the resulting non-cooperative game and show that it performs worse than the centralized algorithm. To overcome this limitation, we introduce a pricing mechanism at the relay, which is shown to achieve the centralized performance. These algorithms, though oblivious to the arrival statistics, do require global knowledge of queue backlogs. Lastly, we consider distributed algorithms that overcome this requirement. Among those, we observe that simple queue-length threshold algorithms perform remarkably well.
\end{abstract}

Index Terms-Cost sharing, delay, network coding, two-way relaying, queue stability, stochastic traffic, cooperation, competition.

\section{INTRODUCTION}

A simple, yet fundamental model for wireless networks is the two-way relay network shown in Fig. 1. In this network, two sources communicate with the assistance of a relay node. Network coding can improve the throughput in this network compared to traditional store-and-forward routing, e.g. [1]. Naturally, the use of the relay will incur a cost, due to, for example, the energy consumption of the relay. Network coding can also reduce this cost [2], [3]. For example, with network coding, the relay can forward one packet from each source simultaneously in a single transmission, while plain routing would require two transmissions [1].

Manuscript received July 28, 2010; revised May 11, 2011; accepted August 22,2011 . The associate editor coordinating the review of this paper and approving it for publication was R. Nabar.

E. N. Ciftcioglu and A. Yener are with the Department of Electrical Engineering, The Pennsylvania State University, University Park, PA 16802, USA (e-mail: enc118@psu.edu, yener@ee.psu.edu).

Y. E. Sagduyu is with Intelligent Automation Inc., Rockville, MD 20855 , USA, and the Institute for Systems Research, University of Maryland, College Park, MD 20742, USA (e-mail: yalinsagduyu@ieee.org).

R. A. Berry is with the Department of Electrical Engineering and Computer Science, Northwestern University, Evanston, IL 60208, USA (e-mail: rberry@eecs.northwestern.edu).

This work was presented in part at the 47th Annual Allerton Conference on Communication, Control, and Computing, September 2009. This work was supported in part by the National Science Foundation under Grant CNS0721445, and the DARPA ITMANET Program under Grant W911NF-07-10028 .

Digital Object Identifier 10.1109/TWC.2011.101211.101360
When the sources have bursty traffic, the cost reduction mentioned above comes at the price of increased delay, since each source must wait for packets to arrive at the other source in order to exploit the network coding gain [4], [5]. This costdelay tradeoff is the main focus of this paper. Specifically, we design transmission policies for two-way communication of bursty sources, which tradeoff the average cost with the average delay. These policies do not require any a priori information of packet arrival statistics and can operate only with the instantaneous values of queue backlogs.

In terms of related work, we note that the energy-delay tradeoff with plain routing has been studied for a single wireless link as well as multiuser settings [6]-[8]. There have been a number of works studying the interaction of network coding with stochastically varying traffic in both two-way relay networks [9]-[11] and other network topologies [12][14]. In all these works, the main focus is on queue stability and the maximum stable throughput without considering transmission costs. Game theoretic models for network coding have been introduced in [15], [16], where the underlying conflict of interest is due to flow optimization rather than cost sharing.

In this paper, we first consider policies in which a centralized controller specifies the transmission rate of each source based on perfect information of both source queue states. We use Lyapunov stability arguments, used in [7], [8], [17] for routing, to develop rate allocation policies for network coding and analyze their cost-delay tradeoff. Next, we consider decentralized operation, where each source independently decides its own rate to minimize its own cost objective, given the joint queue size. This is formulated as a non-cooperative game for which we characterize the equilibrium strategies.

In practice, it is desirable to design algorithms that do not require full knowledge of the queue sizes, i.e., queue state information (QSI), at both sources. We tackle this task by deriving strategies assuming the worst and best response of the opponent thereby, limiting the backlog information to the individual queues only. We then show that dynamic pricing by the relay for the worst-case response can achieve the costoptimal operation at the expense of increasing the complexity of rate allocation.

Next, we present a simple transmission scheme that requires only one bit of QSI from the other source. Specifically, the transmission decisions are made based on whether the individual queue backlogs exceed a fixed threshold. We demonstrate that it is possible to achieve asymptotically optimal cost, as the packet delay grows with increasing threshold values. Finally, we consider the case where sources do not share any QSI. Overall, we observe that the cost-delay tradeoffs obtained from the decentralized rate allocation algorithms proposed in 


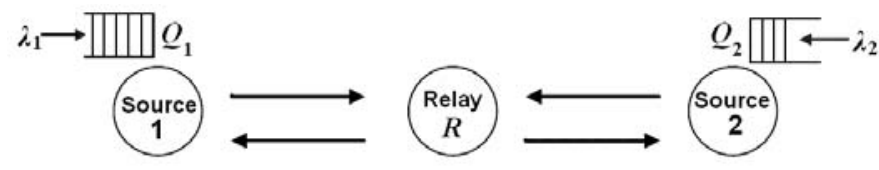

Fig. 1. Two-way relay network: Two sources with packet arrivals and a single relay to exchange the incoming packets.

this paper, approach the cost-delay tradeoff obtained from the centralized algorithm.

The paper is organized as follows. Section II presents the system model, cost structure, and queue dynamics. The centralized and individual cost optimization are considered in Sections III and IV, respectively. We introduce the dynamic pricing policy along with a worst-case response strategy in Section V. This is followed in Section VI by characterizing simple threshold-based rate allocation algorithms. Next, we discuss in Section VII the case when sources have only local queue information. Sections VIII and IX present the performance comparisons of the proposed algorithms and the conclusions, respectively.

\section{SYSTEM MODEL}

We consider a two-way relay network with sources 1 and 2, and relay $R$, as shown in Fig. 1. We assume a slotted system, in which source $i,(i=1,2)$ buffers its incoming packets in queue $Q_{i}$ with backlog $q_{i}(t)$ at time slot $t$. Source $i$ chooses the service rate $\mu_{i}(t)$ at time $t$ such that $\left(\mu_{1}(t), \mu_{2}(t)\right) \in C(t)$, where $C(t)$ is the achievable rate region at time $t$. In this paper, we focus on a simple rate region achievable by network coding:

$$
C(t)=\left\{\left(\mu_{1}(t), \mu_{2}(t)\right): 0 \leq \mu_{i}(t) \leq \mu_{i}^{\max }(t), i=1,2\right\} .
$$

We assume that the two source each communicate with the relay over orthogonal interference-free channels, e.g. separate minislots (TDMA) or separate frequency bands (FDMA). This channel allocation is fixed, resulting in a rectangular rate region for the sources. We adopt this model mainly because of simplicity and to keep the focus of the paper on the effect that network coding has on the cost-delay tradeoff in this twoway information exchange. We assume decode-and-forwardbased digital network coding at the relay. Each packet consists of bits and the relay XOR's two packets, one from each source, componentwise. The model also extends to amplifyand-forward-based analog network coding. The optimization will remain the same except the rate constraints will no longer be modeled by rectangular rate regions. In Section III-A, we will relate this rate region to an underlying physical channel model.

The queue length at source $i, i=1,2$, evolves as

$$
q_{i}(t+1)=\max \left(q_{i}(t)-\mu_{i}(t), 0\right)+a_{i}(t),
$$

where $a_{i}(t)$ is the number of bits/packets arriving at $Q_{i}$ at time $t$. Here, $a_{i}(t)$ is assumed to be an ergodic process with a time-average of $\lambda_{i}$.

Alternatively, the queue dynamics can be written as

$$
q_{i}(t+1)=q_{i}(t)-\tilde{\mu}_{i}(t)+a_{i}(t),
$$

where the transmission rate of source $i$ is

$$
\tilde{\mu}_{i}(t)=\min \left(\mu_{i}(t), q_{i}(t)\right), \quad i=1,2,
$$

since the number of transmitted packets is limited by the number of packets available in the queue. Note that the rate $\mu_{i}(t), i=1,2$, is sufficient to optimize the throughput rates, since sources can simply perform zero padding in the absence of packets. However, we are interested in minimizing the cost, which is a function of the number of transmitted packets. Therefore, we use the transmission rate, $\tilde{\mu}_{i}(t)$, that is bounded by the number packets available in the source queues. The instantaneous rate region is defined as

$$
\begin{aligned}
\tilde{C}(t)= & \left\{\tilde{\mu}_{1}(t), \tilde{\mu}_{2}(t):\right. \\
& \left.\left(\tilde{\mu}_{1}(t), \tilde{\mu}_{2}(t)\right) \in C(t), \tilde{\mu}_{i}(t) \leq q_{i}(t), i=1,2\right\} .
\end{aligned}
$$

We assume that relay $R$ does not buffer the incoming packets and immediately forwards any received data over a channel that is orthogonal to the channels used by each source. The relay decodes and re-encodes the packets incoming from sources before the subsequent transmission in the next time slot. We assume linear network coding in binary field $\mathcal{F}_{2}$. In particular, the relay XOR-es bit streams from an equal number of packets from each source. The remaining residual packets are appended to the XORed bit stream. Then, the relay broadcasts packets back to the sources with rate $\max _{j=1,2} \hat{\mu}_{j}(t)$. Note that the network coding operation can be carried out at packet or signal levels, i.e., the relay either decodes, networkcodes and transmits the incoming packets, or simply amplifiesand-forwards the received signals as in analog network coding [1]. Each source decodes packets by XOR-ing the bit stream received from the relay with the bit stream corresponding to the packets the source transmitted in the previous time slot.

Each use of the relay is assumed to incur a cost, which could, for example, represent the energy or a function of the energy expended by the relay, or an actual cost the relay charges for cooperation. This cost is partitioned among the two sources depending on the rate allocation such that the cost of source $i,(i=1,2)$ for using the relay with rate $\tilde{\mu}_{i}$ is

$$
\begin{aligned}
J_{i}\left(\tilde{\mu}_{1}(t), \tilde{\mu}_{2}(t)\right)= & c_{i}(t)\left(\min _{j=1,2}\left(\tilde{\mu}_{j}(t)\right)\right) \\
& +d_{i}(t)\left(\tilde{\mu}_{i}(t)-\min _{j=1,2}\left(\tilde{\mu}_{j}(t)\right)\right),
\end{aligned}
$$

where $c_{i}(t)$ and $d_{i}(t)$ are the costs charged by the relay to deliver one packet by network coding and routing, respectively. For the sequel, we mainly focus on the case where $c_{i}(t)=\frac{1}{2}$ and $d_{i}(t)=1$, for clarity. This models the case where the cost per unit time of operating the relay is the same for both routing and network coding: in the network coding case the cost is equally shared by both sources, whereas any source is solely responsible for the cost of packets routed by the relay. The individual cost (6) can then be rewritten as

$$
J_{i}\left(\tilde{\mu}_{1}(t), \tilde{\mu}_{2}(t)\right)=\tilde{\mu}_{i}(t)-\frac{1}{2} \min _{j=1,2}\left(\tilde{\mu}_{j}(t)\right)
$$

and the total cost is then simplified to

$J_{t o t}\left(\tilde{\mu}_{1}(t), \tilde{\mu}_{2}(t)\right)=\sum_{i=1}^{2} J_{i}\left(\tilde{\mu}_{1}(t), \tilde{\mu}_{2}(t)\right)=\max _{i=1,2}\left(\tilde{\mu}_{i}(t)\right)$. 
Here, (6)-(8) model linear costs of rate allocation. In Section III-A, we outline the extension to more general rate-cost functions reflecting physical channel characteristics and relate the cost to the needed power for an achievable (informationtheoretic) rate over AWGN channels. We also note that if routing were applied without network coding at the relay, then the cost per packet would be equal to 1, leading to a smaller stability region and larger average delay.

\section{Centralized Optimization}

We start with the case where a centralized controller has access to all system parameters along with the queue backlog information, and makes the transmission decisions for both sources. The main objective is to minimize the total cost for both sources while ensuring that the average delay is no greater than a given value $D$. This leads to the following optimization problem the solution of which will serve as an upper bound on performance of any algorithm that will be considered in the sequel:

\section{Total Minimum Cost Problem (PC):}

$$
\min _{\left(\tilde{\mu}_{1}(t), \tilde{\mu}_{2}(t)\right) \in \tilde{C}(t), t \geq 0} \lim _{t \rightarrow \infty} \frac{1}{t} \sum_{\tau=0}^{t-1} \sum_{i=1}^{2} \mathbb{E}\left[J_{i}\left(\tilde{\mu}_{1}(\tau), \tilde{\mu}_{2}(\tau)\right)\right]
$$

$$
\text { s.t. } \lim _{t \rightarrow \infty} \frac{1}{t} \sum_{\tau=0}^{t-1} \sum_{i=1}^{2} \frac{\mathbb{E}\left[q_{i}(\tau)\right]}{\lambda_{1}+\lambda_{2}}<D .
$$

The average delay constraint follows simply from Little's theorem [18]. The objective in (PC) divided by the total arrival rate $\lambda_{1}+\lambda_{2}$ is equivalent to the long-term average cost per packet. Let $\mathcal{P}^{*}(D)$ denote the solution to (PC) as a function of the delay constraint $D$, i.e., the cost-delay tradeoff. $\mathcal{P}^{*}(D)$ is a decreasing function of $D$ and as $D \rightarrow \infty$, it will yield the minimum cost solution subject to the queue's being stable.

In principle, for a given delay constraint, (PC) can be solved via dynamic programming. However, such a solution quickly becomes intractable except for very simple arrival processes and furthermore, requires a priori knowledge of arrival statistics. Instead, we will follow the approach in [8] and use Lyapunov stability arguments to yield an approximate solution to $(\mathbf{P C})$ with provable approximation bounds. This approach is based on generalizing the classical back-pressure algorithm from [17] which is throughput-optimal, i.e., it stabilizes the queues whenever possible. Specifically, at each time $t$, the back-pressure algorithm solves

$$
\max _{\left(\mu_{1}(t), \mu_{2}(t)\right) \in C(t), t \geq 0} \sum_{i=1}^{2} q_{i}(t) \mu_{i}(t),
$$

or equivalently

$$
\max _{\left(\tilde{\mu}_{1}(t), \tilde{\mu}_{2}(t)\right) \in \tilde{C}(t), t \geq 0} \sum_{i=1}^{2} q_{i}(t) \tilde{\mu}_{i}(t) .
$$

Observe that (9) (or (10)) does not necessarily yield an optimal policy for (PC). Consider, for instance, the following example. Let the number of packet arrivals be $a_{1}(t)=1$, $a_{2}(t)=2$ for even $t$, and $a_{1}(t)=2, a_{2}(t)=1$ for odd $t$. For $c_{i}(t)=\frac{1}{2}$ and $d_{i}(t)=1$, the back-pressure solution (9)

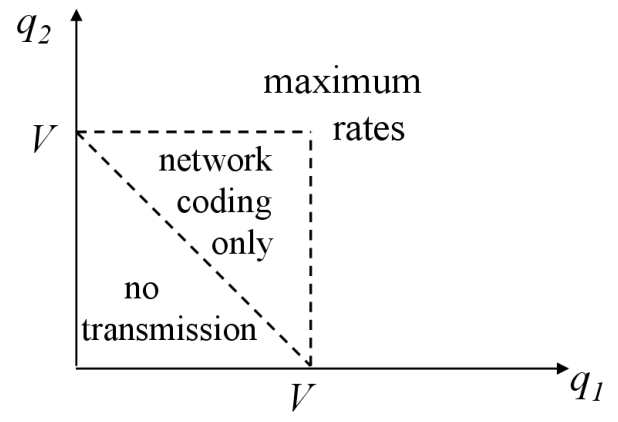

Fig. 2. Centralized solution, (A1), for Source 1, Source 2 transmissions.

or (10) is $\mu_{1}(t)=1, \mu_{2}(t)=2$ for even $t$, and $\mu_{1}(t)=2$, $\mu_{2}(t)=1$ for odd $t$. The long-term average costs per packet $J_{1}$ and $J_{2}$ are $\frac{2}{3}$. However, if sources accumulate packets over time slots and transmit such that relay $R$ performs network coding only, the decisions $\mu_{1}(t)=1, \mu_{2}(t)=1$ for even $t$ and $\mu_{1}(t)=2, \mu_{2}(t)=2$ for odd $t$ result in the long-term average costs approaching the lower bound $\frac{1}{2}$. This is attainable, if the relay performs network coding with the cost for each relay transmission equally shared between the sources.

Fortunately, [8] provides a way to augment the policy in (10) with an additional term to reflect the cost. Adopting this approach, our modified problem becomes

$\max _{\left(\tilde{\mu}_{1}(t), \tilde{\mu}_{2}(t)\right) \in \tilde{C}(t)}\left(q_{1}(t) \tilde{\mu}_{1}(t)+q_{2}(t) \tilde{\mu}_{2}(t)-V \max \left(\tilde{\mu}_{1}(t), \tilde{\mu}_{2}(t)\right)\right)$,

where the control parameter $V$ tunes the tradeoff between the average queue backlog and the distance from the minimum cost. In (P1), maximizing the term $q_{1}(t) \tilde{\mu}_{1}(t)+q_{2}(t) \tilde{\mu}_{2}(t)$ is due to backpressure algorithm stabilizing the source queues, whereas minimizing the term $\max \left(\tilde{\mu}_{1}(t), \tilde{\mu}_{2}(t)\right)$ moves the stable solution to the minimum cost. Next, we present this solution.

Theorem 1: At time slot $t$, the solution to (P1) is given by

$\left(\tilde{\mu}_{i}(t), \tilde{\mu}_{j}(t)\right)=$

$\begin{cases}(0,0), & \text { if } V>q_{1}(t)+q_{2}(t), \\ \left(u_{c}(t), u_{c}(t)\right), & \text { if } q_{1}(t)+q_{2}(t)>V \geq \max \left(q_{1}(t), q_{2}(t)\right), \\ \left(u_{i}(t), u_{c}(t)\right), & \text { if } q_{i}(t)>V \geq q_{j}(t), j \neq i, \\ \left(u_{i}(t), u_{c}(t)\right), & \text { if } V \leq \min \left(q_{1}(t), q_{2}(t)\right), \\ & \left(q_{i}(t)-V\right) u_{i}(t)+q_{j}(t) u_{c}(t) \\ & \geq\left(q_{j}(t)-V\right) u_{j}(t)+q_{i}(t) u_{c}(t), j \neq i,\end{cases}$

where

$u_{i}(t)=\min \left(q_{i}(t), \mu_{i}^{\max }(t)\right), \quad u_{c}(t)=\min \left(u_{1}(t), u_{2}(t)\right)$.

Transmission policy (A1) is depicted in Figure 2 for clarity.

Proof: The objective function depends on which source is assigned a higher rate, and it is either equal to $\left(q_{1}(t)-\right.$ $V) \tilde{\mu}_{1}(t)+q_{2}(t) \tilde{\mu}_{2}(t)$, if $\tilde{\mu}_{1}(t) \geq \tilde{\mu}_{2}(t)$ or $\left(q_{2}(t)-V\right) \tilde{\mu}_{2}(t)+$ $q_{1}(t) \tilde{\mu}_{1}(t)$, if $\tilde{\mu}_{2}(t) \geq \tilde{\mu}_{1}(t)$. Consider the case with $q_{i}(t)>V$ and $q_{j}(t)<V$. For $\tilde{\mu}_{i}(t) \geq \tilde{\mu}_{j}(t)$, the objective function is greater than or equal to $\left(q_{1}(t)+q_{2}(t)-V\right) u_{c}(t)$, whereas the objective function is at most $\left(q_{1}(t)+q_{2}(t)-V\right) u_{c}(t)$ for $\tilde{\mu}_{j}(t) \geq \tilde{\mu}_{i}(t)$. Accordingly, the rate allocation with $\mu_{i}(t) \geq$ $\mu_{j}(t)$ is selected with $\tilde{\mu}_{i}(t)$ equal to $u_{i}(t)$ and $\tilde{\mu}_{j}(t)$ equal to 
$u_{c}(t)$.

Without loss of generality, consider the case with $\tilde{\mu}_{i}(t) \geq$ $\tilde{\mu}_{j}(t)$. Then, $\max \left(\tilde{\mu}_{1}(t), \tilde{\mu}_{2}(t)\right)=\tilde{\mu}_{i}(t)$, and the objective function is $\left(q_{i}(t)-V\right) \tilde{\mu}_{i}(t)+q_{j}(t) \tilde{\mu}_{j}(t)$.

If $q_{i}(t)<V$, the optimal solution should minimize $u_{i}(t)$, since the coefficient associated with the rate of source $i$ is negative. However, the special case with $\tilde{\mu}_{i}(t)=\tilde{\mu}_{j}(t)$ should be also taken into account. If $q_{j}(t)>\left|q_{i}(t)-V\right|$, or equivalently if $q_{1}(t)+q_{2}(t)>V$, then the optimal rate allocation is $\tilde{\mu}_{i}(t)=\tilde{\mu}_{j}(t)=u_{c}(t)$. On the other hand, if $q_{j}(t)<\left|q_{i}(t)-V\right|$, i.e., if $q_{1}(t)+q_{2}(t)<V$, the optimal rate allocation is $\tilde{\mu}_{i}(t)=\tilde{\mu}_{j}(t)=0$.

Finally, if both $q_{i}(t)$ and $q_{j}(t)$ exceed $V$, rates should be selected from one of the two possibilities in the fourth case of (A1) to maximize the overall objective function.

We next present the performance of (A1).

Theorem 2: For Algorithm (A1), the average queue back$\log$ and the average packet delay are proportional to $V$, while the distance of the average cost from the minimum attainable cost is proportional to $\frac{1}{V}$, i.e.,

$$
\begin{aligned}
& \lim _{M \rightarrow \infty} \frac{1}{M} \sum_{\tau=0}^{M-1} \sum_{i=1}^{2} J_{i}\left(\tilde{\mu}_{1}(\tau), \tilde{\mu}_{2}(\tau)\right) \leq J^{*}+\frac{B_{1}}{V}, \\
& \lim _{M \rightarrow \infty} \frac{1}{M} \sum_{\tau=0}^{M-1} \sum_{i=1}^{2} \mathbb{E}\left[q_{i}(\tau)\right] \leq \frac{B_{2}+\frac{V}{2}}{\epsilon}
\end{aligned}
$$

where $J^{*}$ is the optimal cost per time slot, $B_{1}$ and $B_{2}$ are positive constants, and $\lambda_{i}$ satisfies $\left(\lambda_{i}+\epsilon\right) \in C(t), i=1,2$, at any time $t$ for an arbitrarily small positive constant $\epsilon$.

Proof: We provide a sketch of the proof for brevity. We use the Lyapunov drift analysis from [8]. First, the Lyapunov drift, namely, the time-average difference of Lyapunov functions for queue backlogs is formulated using the queue dynamics along with the average cost. Stability is guaranteed whenever the drift is negative. Next, we show that (A1) satisfies this condition. This way, we first find the upper bound on the average queue length and then use Little's theorem to derive the upper bound on the average delay and cost.

Both problems (PC) and (P1) stabilize queues and provide bounded delay. While this bound is explicit in (PC), the solution of (P1) provides an indirect delay bound that follows from queue stabilization. To relate $(\mathbf{P C})$ with $(\mathbf{P 1})$, we first note that $J^{*}$ in Theorem 2 is equal to $\mathcal{P}(D)$ as $D \rightarrow \infty$. Second, we can specify a common delay upper bound $D$ through the relation (12):

$$
D \leq \frac{B_{2}+\frac{V}{2}}{\epsilon\left(\lambda_{1}+\lambda_{2}\right)}
$$

which yields $V \geq 2\left(D \epsilon\left(\lambda_{1}+\lambda_{2}\right)-B_{2}\right)$. Using a parameter $V$ accordingly in (11), we could bound the RHS of (11) as

$$
J^{*}+\frac{B_{1}}{V} \leq J^{*}+\frac{B_{1}}{2\left(D \epsilon\left(\lambda_{1}+\lambda_{2}\right)-B_{2}\right)} .
$$

This puts an upper bound on the cost performance of (P1) and guarantees delay performance of $D$. On the other hand, (PC) achieves the cost performance $\mathcal{P}(D)$, which is known to be greater than $J^{*}$ for any finite average delay requirement $D$. As a result, Theorem 2 also bounds the distance between the costs attained by solving (P1) compared with the solution of (PC). We note that while the delay bound in $(\mathbf{P C})$ is tight in general, the bounds provided in (11)-(12) for the cost and delay performance of (P1) are not necessarily tight. Our goal is to characterize this interplay between cost and delay, and evaluate the performance through feasible algorithms with tractable analysis. The upper bounds on delay characterize the worst-case performance and highlights the robustness of the practical approximate algorithms.

For (P1) the optimal cost per packet $J_{p}^{o p t}=\frac{J^{*}}{\lambda_{1}+\lambda_{2}}$ is given by $\frac{\max \left(\lambda_{1}, \lambda_{2}\right)}{\lambda_{1}+\lambda_{2}}$, and it is reduced to $\frac{1}{2}$ for $\lambda_{1}=\lambda_{2}$. For $q_{1}(t)+q_{2}(t)>V>\max \left(q_{1}(t), q_{2}(t)\right)$, the relay $R$ performs network coding only and does not forward uncoded packets. This results in energy-optimal operation. As $V$ increases, the sources tend to transmit less, and the relay finds more opportunities for coded transmissions. As $V$ decreases, the relay forwards more uncoded traffic, and the policy resembles more and more the classical back-pressure algorithm.

\section{A. General Costs based on Physical Channel Properties}

Next, we generalize the linear cost function in (8) by relating the achievable rates to energy. The relay communication consists of two phases: Multiple access from sources to the relay, and broadcast from the relay to sources. We assume that the achievable rates in the first phase are larger than those in the second phase thereby imposing the bottleneck links. We assume additive white Gaussian noise channels with unit noise power and bandwidth. In the broadcast phase, the individual min-cut capacity for source $i, i=1,2$, can be achieved via XOR-based network coding [19]. At time slot $t$, the end-toend rate from source $i$ forwarded by the relay satisfies

$$
\mu_{i}(t) \leq \log \left(1+h_{j} P(t)\right),
$$

where $\sqrt{h_{j}}$ is the constant channel gain from the relay to source $j, j \neq i$, and $P(t)$ is the transmission power of either source. The relay power is chosen so as to satisfy the signal-tonoise-ratio (SNR) requirement at both receivers. Let $\gamma_{i}=h_{j} P$ denote the SNR from source $i$ to source $j$ with unit noise power and bandwidth. For low SNR, we have $\log (1+\gamma) \approx \gamma$ and there is a linear relationship between the rate and the power, as we assumed so far. For high SNR, i.e., $\gamma \gg 1$, $\log (1+\gamma) \approx \log \gamma$, and the power is exponential function of the rate supported.

We generalize the cost functions (6)-(8) to the case with asymmetric channel gains between the relay and sources. For low SNR, the total and individual costs for $i \neq j, i=1,2$, are

$$
\begin{aligned}
J_{t o t}\left(\tilde{\mu}_{1}(t), \tilde{\mu}_{2}(t)\right) & =\max \left(\frac{\tilde{\mu}_{1}(t)}{h_{2}}, \frac{\tilde{\mu}_{2}(t)}{h_{1}}\right), \\
J_{i}\left(\tilde{\mu}_{1}(t), \tilde{\mu}_{2}(t)\right) & =\frac{\tilde{\mu}_{i}(t)}{h_{j}}-\frac{1}{2} \min \left(\frac{\tilde{\mu}_{1}(t)}{h_{2}}, \frac{\tilde{\mu}_{2}(t)}{h_{1}}\right) .
\end{aligned}
$$

The centralized solution for (PC) with (16) as the cost is given by the algorithm $(\mathbf{A 1 a})$ : 


$$
\begin{aligned}
& \left(\tilde{\mu}_{i}(t), \tilde{\mu}_{j}(t)\right)= \\
& \begin{cases}(0,0), & \text { if } \min \left(\frac{V}{h_{1}}, \frac{V}{h_{2}}\right)>q_{1}(t)+q_{2}(t), \\
\left(u_{i}(t), u_{i}(t) \frac{h_{i}}{h_{j}}\right), & \text { if } h_{i} u_{i}(t) \geq h_{j} u_{j}(t), j \neq i, \\
& \left(q_{1}(t)+q_{2}(t)\right) \max \left(h_{1}, h_{2}\right)>V, \\
& V \geq \max \left(q_{1}(t) h_{2}, q_{2}(t) h_{1}\right), \\
\left(u_{i}(t), u_{c j}(t)\right), & \text { if } q_{i}(t) h_{j}>V \geq q_{j}(t) h_{i}, j \neq i, \\
\left(u_{i}(t), u_{c j}(t)\right), & \text { if } V \leq \min \left(q_{1}(t) h_{2}, q_{2}(t) h_{1}\right), \\
& \left(q_{i}(t)-V\right) u_{i}(t)+q_{j}(t) u_{c j}(t) \\
& \geq\left(q_{j}(t)-V\right) u_{j}(t)+q_{i}(t) u_{c i}(t), j \neq i,\end{cases}
\end{aligned}
$$

where

$$
\begin{aligned}
u_{i}(t) & =\min \left(q_{i}(t), \mu_{i}^{\max }(t)\right), \\
u_{c j}(t) & =\min \left(u_{1}(t), u_{2}(t), u_{i}(t) \frac{h_{i}}{h_{j}}\right) .
\end{aligned}
$$

For the high SNR regime, the total and individual costs functions for $i \neq j, i=1,2$, are

$$
\begin{aligned}
J_{t o t}\left(\tilde{\mu}_{1}(t), \tilde{\mu}_{2}(t)\right) & =\max \left(2^{\tilde{\mu}_{1}(t)}, 2^{\tilde{\mu}_{2}(t)}\right) \\
J_{i}\left(\tilde{\mu}_{1}(t), \tilde{\mu}_{2}(t)\right) & =2^{\tilde{\mu}_{i}(t)}-\frac{1}{2} \min \left(2^{\tilde{\mu}_{1}(t)}, 2^{\tilde{\mu}_{2}(t)}\right)(21)
\end{aligned}
$$

for the case with symmetric channels. Then, at any time slot $t$, the centralized solution for (PC) with (20) as the cost function is given by the algorithm (A1b) as follows:

$$
\begin{aligned}
& \left(\tilde{\mu}_{i}(t), \tilde{\mu}_{j}(t)\right)= \\
& \left\{\begin{array}{l}
(0,0), \\
\left(u_{n c}(t), u_{n c}(t)\right), \\
\left(r_{i}(t), u_{c j}(t)\right), \\
\left(r_{i}(t), u_{c j}(t)\right),
\end{array}\right.
\end{aligned}
$$

$$
\begin{aligned}
& \text { if } V>q_{1}(t)+q_{2}(t), \\
& \text { if } q_{1}(t)+q_{2}(t)>V, \\
& u_{n c}(t) \leq \min \left(u_{1}(t), u_{2}(t)\right), \\
& \text { if } q_{i}(t)>V \geq q_{j}(t), \\
& j \neq i, u_{n c}(t)>\min \left(u_{1}(t), u_{2}(t)\right), \\
& \text { if } V \leq \min \left(q_{1}(t), q_{2}(t)\right), \\
& u_{n c}(t)>\min \left(u_{1}(t), u_{2}(t)\right), \\
& q_{i}(t) r_{i}(t)-V 2^{r_{i}(t)}+q_{j}(t) u_{c j}(t) \\
& \geq q_{j}(t) r_{j}(t)-V 2^{r_{j}(t)}+q_{i}(t) u_{c i}(t), j \neq i,
\end{aligned}
$$

(A1b)

where

$$
\begin{aligned}
u_{i}(t) & =\min \left(q_{i}(t), \mu_{i}^{\max }(t)\right), \\
r_{i}(t) & =\min \left(u_{i}(t), \log \left(\frac{q_{i}(t)}{V}\right)\right), \\
u_{c j}(t) & =\min \left(r_{i}(t), u_{j}(t)\right), \\
u_{n c}(t) & =\log \left(\frac{q_{1}(t)+q_{2}(t)}{V}\right) .
\end{aligned}
$$

Observe that the algorithm above works more aggressively compared with (A1a) to equalize the rates because of the exponential relation between the power and achievable rate from (20).

\section{INDIVIDUAL COST OPTIMIZATION}

Although in the previous section we have shown that our approach holds for general cost functions, e.g. (20), in the sequel, we will use linear cost functions, i.e., (7), for clarity.
Next, we consider a scenario in which the individual nodes decide on their own rate allocations given perfect QSI of the other user. Each user $i=1,2$ solves the following optimization problem:

\section{Individual Minimum Cost Problem (PI):}

$$
\min _{\tilde{\mu}_{i}(t):\left(\tilde{\mu}_{1}(t), \tilde{\mu}_{2}(t)\right) \in \tilde{C}(t), t \geq 0} \lim _{t \rightarrow \infty} \frac{1}{t} \sum_{\tau=0}^{t-1} \mathbb{E}\left[J_{i}\left(\left(\tilde{\mu}_{1}(\tau), \tilde{\mu}_{2}(\tau)\right)\right]\right.
$$

$$
\text { s.t. } \lim _{t \rightarrow \infty} \frac{1}{t} \sum_{\tau=0}^{t-1} \frac{\mathbb{E}\left[q_{i}(\tau)\right]}{\lambda_{i}}<D_{i} \text {. }
$$

The two users can be viewed as players in a non-cooperative game, in which (PI) reflects the pay-off of user $i$. In principle, this can be viewed as a stochastic game [20] and can be solved via dynamic programming. However, once again such an approach quickly becomes intractable. We instead consider a Lyapunov drift formulation as in (P1). Furthermore, we assume that in each time-slot, sources play a single-stage game against each other with such that given $\tilde{\mu}_{j}, j \neq i$, source $i=1,2$ chooses the individual strategy $\tilde{\mu}_{i}(t)$ to solve:

$$
\max _{\tilde{\mu}_{i}(t):\left(\tilde{\mu}_{1}(t), \tilde{\mu}_{2}(t)\right) \in \tilde{C}(t), t \geq 0}\left(q_{i}(t) \tilde{\mu}_{i}(t)-V_{i} J_{i}\left(\tilde{\mu}_{1}(t), \tilde{\mu}_{2}(t)\right)\right) .
$$

This formulation does not require any knowledge of the long-term arrival statistics of either source. Minimizing the first term $q_{i}(t) \tilde{\mu}_{i}(t)$ in the individual objective function is to stabilize the queue of source $i$, whereas minimizing the second term $V_{i} J_{i}\left(\tilde{\mu}_{1}(t), \tilde{\mu}_{2}(t)\right)$ moves the stable solution to the optimal cost value. (P1) is based on cooperation of sources to optimize the system performance of stabilizing both queues and minimizing the total cost. On the other hand, (P2) assumes that sources do not cooperate with each other but rather optimize their individual performance objectives. In particular, in (P2) sources are unaware of each other's transmission strategies. Compared to (P1) this lack of coordination results in total performance loss, which we will evaluate in Section VIII. Define $\tilde{J}_{i}\left(\tilde{\mu}_{1}(t), \tilde{\mu}_{2}(t)\right)=q_{i}(t) \tilde{\mu}_{i}(t)-V_{i} J_{i}\left(\tilde{\mu}_{1}(t), \tilde{\mu}_{2}(t)\right)$.

Definition 1: A pair of strategies $\left\{\tilde{\mu}_{i}^{*}\right\}$ are a Nash equilibrium if

$$
\tilde{J}_{i}\left(\tilde{\mu}_{i}^{*}, \tilde{\mu}_{-i}^{*}\right) \geq \tilde{J}_{i}\left(\tilde{\mu}_{i}, \tilde{\mu}_{-i}^{*}\right), \text { for all } \tilde{\mu}_{i} \in \tilde{C}(t)
$$

for each player $i$, where " $-i$ " denotes the player other than player $i$.

For any source $i=1,2$, the strategy space is a non-empty convex subset of Euclidean space and the utility function is continuous in $\left(\tilde{\mu}_{1}, \tilde{\mu}_{2}\right)$ and quasi-concave in $\tilde{\mu}_{i}$. Therefore, there exists a pure strategy Nash equilibrium at any time slot $t$ for given $q_{i}(t), i=1,2$.

Theorem 3: At time slot $t$, the solution to (P2) is given by the Nash equilibrium strategy (A2) for source $i=1,2$. (A2) 


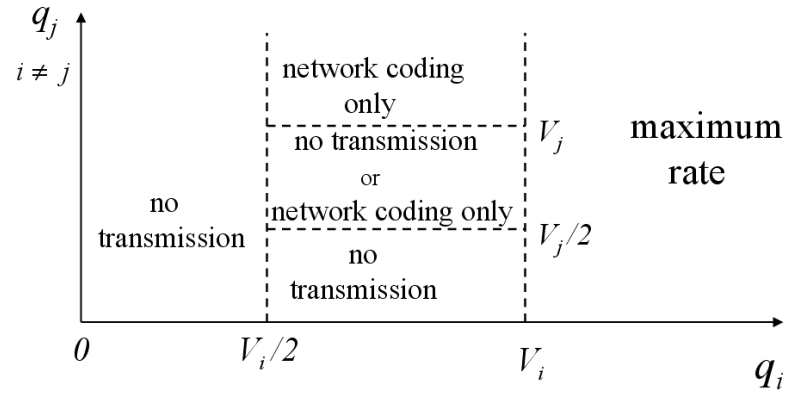

Fig. 3. Individual solution, (A2), for Source $i . i, j=1,2$.

is depicted in Figure 3.

$$
\tilde{\mu}_{i}(t)= \begin{cases}0, & \text { if } q_{i}(t)<\frac{V_{i}}{2}, \\ u_{i}(t), & \text { if } q_{i}(t) \geq V_{i}, \\ 0, & \text { if } \frac{V_{i}}{2} \leq q_{i}(t)<V_{i}, q_{j}(t)<\frac{V_{j}}{2}, j \neq i, \\ u_{c}(t), & \text { if } \frac{V_{i}}{2} \leq q_{i}(t)<V_{i}, q_{j}(t) \geq V_{j}, j \neq i, \\ 0 \text { or } u_{c}(t), & \text { if } \frac{V_{i}}{2} \leq q_{i}(t)<V_{i}, i=1,2 .\end{cases}
$$

Proof: Note that $J_{i}\left(\tilde{\mu}_{1}(t), \tilde{\mu}_{2}(t)\right)=\tilde{\mu}_{i}(t)-$ $\frac{1}{2} \min _{j=1,2}\left(\tilde{\mu}_{j}(t)\right)$ is equal to $\tilde{\mu}_{i}(t)-\frac{1}{2} \tilde{\mu}_{j}(t)$, if $\tilde{\mu}_{i}(t) \geq$ $\tilde{\mu}_{j}(t)$, or equal to $\frac{1}{2} \tilde{\mu}_{i}(t)$, if $\tilde{\mu}_{j}(t) \geq \tilde{\mu}_{i}(t)$. Without loss of generality, consider the former case, in which the objective function is $\left(q_{i}(t)-V_{i}\right) \tilde{\mu}_{i}(t)+\frac{V_{i}}{2} q_{j}(t) \tilde{\mu}_{j}(t)$ for source $i$, and $\left(q_{j}(t)-\frac{V_{j}}{2}\right) \tilde{\mu}_{j}(t)$ for source $j \neq i$. The latter case follows from interchanging $i$ and $j$. If $q_{i}(t)>V_{i}, i=1,2$, the largest possible $\tilde{\mu}_{i}(t)$ is selected as $u_{i}(t)$, since the coefficient of source $i$ 's rate will be always positive. Similarly, if $q_{i}(t)<\frac{V_{i}}{2}$, $i=1,2$, the smallest possible $\tilde{\mu}_{i}(t)$ is selected as 0 , since the coefficient of source $i$ 's rate will be always negative.

Consider $\frac{V_{i}}{2}<q_{i}(t)<V_{i}$. If $q_{j}(t)<\frac{V_{j}}{2}$, source $j$ will not transmit and the best strategy of source $i$ is to reduce $u_{i}(t)$ to 0 . If $q_{j}(t)>V_{j}$, the best strategy of source $j$ is to transmit. Then, source $i$ either tends to decrease its rate to $u_{c}(t)$ for $\tilde{\mu}_{i}(t) \geq \tilde{\mu}_{j}(t)$, or tends to increase its rate to $u_{c}(t)$ for $\tilde{\mu}_{j}(t) \geq \tilde{\mu}_{i}(t)$. Source $i$ selects the rate $u_{c}(t)$ for either case. For $\frac{V_{i}}{2}<q_{i}(t)<V_{i}, i=1,2$, there are multiple Nash equilibria including $\mu_{i}(t)=0$ and $\mu_{i}(t)=u_{c}(t), i=1,2$.

\section{Dynamic Pricing at the Relay}

We now consider the case where sources have no information about each other's queue backlog in contrast to $(\mathbf{P} 2)$. Thus source $i$ assumes that the opponent source $j \neq i$ plays a fixed strategy. The worst-case response of the opponent $j$ is $\tilde{\mu}_{j}=0$ such that there is no possibility of network coding to reduce the relay cost. If source $i=1,2$ assumes that source $j \neq i$ plays the worst-case strategy, it ends up with the optimization problem:

$$
\max _{\tilde{\mu}_{i}(t):\left(\tilde{\mu}_{i}(t), 0\right) \in \tilde{C}(t), t \geq 0}\left(q_{i}(t) \tilde{\mu}_{i}(t)-V_{i} J_{i}\left(\tilde{\mu}_{i}(t), 0\right)\right) .
$$

The solution to $(\mathbf{P 3})$ is given by

$$
\tilde{\mu}_{i}(t)= \begin{cases}0, & \text { if } q_{i}(t)<V_{i}, \\ u_{i}(t), & \text { if } q_{i}(t) \geq V_{i} .\end{cases}
$$

As shown in Figure 4, (A3) simply compares the individual queue backlog with a threshold that is independent of the

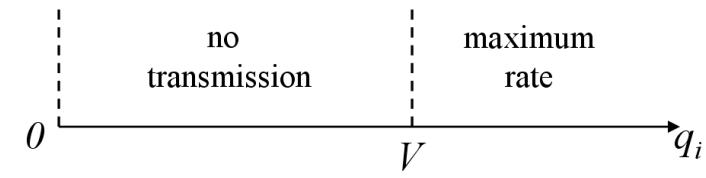

Fig. 4. Solution by playing Worst Case response, (A3), for Source $i . i=1,2$.

opponent's queue backlog or transmission decision. A similar algorithm can be defined by assuming that the opponent $j$ plays the best-case strategy, which is $\tilde{\mu}_{j}=\mu_{j}^{\max }(t)$ so that packets of source $i$ can always be network-coded. The resulting problem is

$\max _{\tilde{\mu}_{i}(t):\left(\tilde{\mu}_{i}(t), \mu_{j}^{\max }(t)\right) \in C(t), j \neq i}\left(q_{i}(t) \tilde{\mu}_{i}(t)-V_{i} J_{i}\left(\tilde{\mu}_{i}(t), \mu_{j}^{\max }(t)\right)\right)$.

The solution (A4) to (P4) is given by:

$\tilde{\mu}_{i}(t)=\left\{\begin{array}{l}0 \\ \mu_{j}^{\max }(t) \\ u_{i}(t) \\ u_{i}(t)\end{array}\right.$

$$
\begin{aligned}
& \text { if } q_{i}(t)<\frac{V_{i}}{2}, \\
& \text { if } u_{i}(t) \geq \mu_{j}^{\max }(t), \\
& \frac{V_{i}}{2} \leq q_{i}(t)<V_{i}, j \neq i, \\
& \text { if } u_{i}(t) \geq \mu_{j}^{\max }(t), q_{i}(t) \geq V_{i}, j \neq i, \\
& \text { if } u_{i}(t)<\mu_{j}^{\max }(t), q_{i}(t) \geq \frac{V_{i}}{2}, j \neq i .
\end{aligned}
$$

So far, we assumed fixed weights $c_{i}(t)=\frac{1}{2}$ for network coding and $d_{i}(t)=1$ for routing. Instead, suppose the relay uses dynamic pricing by selecting the weights $c_{i}(t)$ and $d_{i}(t)$ in (6) as functions of queue backlogs $q_{1}(t)$ and $q_{2}(t)$ to adapt the distributed energy costs to the centralized optimal solutions. For that purpose, relay penalizes the difference between the data rates transmitted from each source thereby increasing the network coding opportunities and minimizing the relay cost. The individual cost for node $i$ is changed to

$$
\begin{aligned}
& J_{i}\left(\tilde{\mu}_{1}(t), \tilde{\mu}_{2}(t)\right)=c_{i}(t) \min _{j=1,2} \tilde{\mu}_{j}(t)+d_{i}(t) \\
& \left.\quad \times\left(\tilde{\mu}_{i}(t)-\min _{j=1,2} \tilde{\mu}_{j}(t)\right)+e_{i}(t) \max _{j=1,2} \tilde{\mu}_{j}(t)-\min _{j=1,2} \tilde{\mu}_{j}(t)\right),
\end{aligned}
$$

where $e_{i}(t)$ is an additional weight to equalize the transmission rates for both sources. The individual optimization problem is then given by

$$
\begin{aligned}
& \max _{\tilde{\mu}_{i}(t):\left(\tilde{\mu}_{i}(t)\right) \in C(t), t \geq 0}\left(q_{i}(t) \tilde{\mu}_{i}(t)-d_{i}(t) \tilde{\mu}_{i}(t)\right. \\
& \left.+\left(d_{i}(t)+e_{i}(t)-c_{i}(t)\right) \min _{j=1,2} \tilde{\mu}_{j}(t)-e_{i}(t) \max _{j=1,2} \tilde{\mu}_{j}(t)\right),
\end{aligned}
$$

where we again assume the sources play the worst-case response without knowing each other's queue backlogs. In particular, we do not require that the weights are globally known, i.e. they can be delivered only to the respective sources.

Theorem 4: Given the queue backlogs $q_{i}(t), i=1,2$, and threshold parameter $V$, the rate weights $c_{i}(t), d_{i}(t)$ and $e_{i}(t)$ for (A3) to achieve the optimal solutions (A1) satisfy:

$$
\begin{aligned}
& \text { If } V>q_{1}(t)+q_{2}(t): q_{i}(t)<c_{i}(t)<d_{i}(t)+e_{i}(t), \\
& \quad i=1,2 . \\
& \text { If } q_{1}(t)+q_{2}(t) \geq V>\max \left(q_{1}(t), q_{2}(t)\right): \\
& \quad d_{i}(t)+e_{i}(t)>q_{i}(t), c_{i}(t)>d_{i}(t)+e_{i}(t), \\
& \quad i=1,2 .
\end{aligned}
$$




$$
\begin{aligned}
& \text { If } q_{i}(t) \geq V>q_{j}(t), j \neq i: d_{i}(t)+e_{i}(t)<q_{i}(t) \\
& \quad d_{j}(t)+e_{j}(t)>q_{j}(t), c_{j}(t)>d_{j}(t)+e_{j}(t) \\
& \text { If } V \leq \min \left(q_{1}(t), q_{2}(t)\right), \quad\left(q_{i}(t)-V\right) u_{i}(t)+q_{j}(t) u_{c}(t) \\
& \quad \geq\left(q_{j}(t)-V\right) u_{j}(t)+q_{i}(t) u_{c}(t), j \neq i: \\
& \quad d_{i}(t)+e_{i}(t)<q_{i}(t), d_{j}(t)+e_{j}(t)>q_{j}(t) \\
& \quad c_{j}(t)>d_{j}(t)+e_{j}(t) .
\end{aligned}
$$

Proof: We arrange the coefficients in (28) and adjust the weights $c_{i}(t), d_{i}(t)$ and $e_{i}(t)$ to mimic the behavior of the centralized case (A1). The objective function depends on which source is assigned a higher rate, and is equal to $\left(q_{i}(t)-d_{i}(t)-e_{i}(t)\right) \tilde{\mu}_{i}(t)+\left(d_{i}(t)+e_{i}(t)-c_{i}(t)\right) \tilde{\mu}_{j}(t)$, if $\tilde{\mu}_{i}(t) \geq \tilde{\mu}_{j}(t)$, or $\left(q_{i}(t)+e_{i}(t)-c_{i}(t)\right) \tilde{\mu}_{i}(t)-e_{i}(t) \tilde{\mu}_{j}(t)$, if $\tilde{\mu}_{i}(t) \leq \tilde{\mu}_{j}(t)$. For brevity, we will consider two subcases. First, assume $V>q_{1}(t)+q_{2}(t)$, where the solution should be $\tilde{\mu}_{j}(t)=\tilde{\mu}_{i}(t)=0$ from (A1). If $c_{i}(t)<d_{i}(t)+e_{i}(t)$, assuming $\tilde{\mu}_{i}(t) \geq \tilde{\mu}_{j}(t)$, the worst-case response is $\tilde{\mu}_{j}(t)=0$, and if $d_{i}(t)+e_{i}(t)>q_{i}(t), \tilde{\mu}_{i}(t)$ is selected as 0 , resulting in an objective value of 0 . For $\tilde{\mu}_{i}(t) \leq \tilde{\mu}_{j}(t)$, the worst case response is $\tilde{\mu}_{j}(t)=0$ for $e_{i}(t)<0$ and $\tilde{\mu}_{j}(t)=$ $u_{j}(t)$ if $e_{i}(t)>0$. For $e_{i}(t)<0$, the desired solution is $\tilde{\mu}_{i}(t)=0$ from the initial assumption $\tilde{\mu}_{i}(t) \leq \tilde{\mu}_{j}(t)$. For $e_{i}(t)>0$, the maximum value of the objective function is $\left(q_{i}(t)+e_{i}(t)-c_{i}(t)\right) u_{c}(t)-e_{i}(t) u_{c}(t)=\left(q_{i}(t)-c_{i}(t)\right) u_{c}(t)$. With $q_{i}(t)<c_{i}(t)$, the objective value is negative and the overall rate selection is $\tilde{\mu}_{i}(t)=0$. Changing the indices $i$ and $j$ results in the symmetric solutions.

Next, assume $q_{1}(t)+q_{2}(t)>V>\max \left(q_{1}(t), q_{2}(t)\right)$. For $\tilde{\mu}_{i}(t) \geq \tilde{\mu}_{j}(t)$, by selecting $c_{i}(t)>d_{i}(t)+e_{i}(t)$, the worst-case response is to maximize $\tilde{\mu}_{j}(t)$. However, if $d_{i}(t)+e_{i}(t)>q_{i}(t)$, source $i$ tends to reduce $\tilde{\mu}_{i}(t)$ to $u_{c}(t)$ because of the worst-case response from source $j$. Then, the objective function is $\left(q_{i}(t)-c_{i}(t)\right) u_{c}(t)$. The maximum objective value achieved by assuming $\tilde{\mu}_{i}(t) \leq \tilde{\mu}_{j}(t)$ is also equal to $\left(q_{i}(t)-c_{i}(t)\right) u_{c}(t)$, with $\tilde{\mu}_{i}(t)=u_{c}(t)$. Hence, the overall solution is $\tilde{\mu}_{i}(t)=u_{c}(t)$. The other two subcases for (A1) are derived similarly. By setting $d_{i}(t)+e_{i}(t)<q_{i}(t)$, the rates of source $i$ are increased in accordance with the solution of (A1).

\section{Cost Sharing With 1-Bit Queue Information}

For the individual cost optimization problem, we can further simplify the transmission decisions by limiting the necessary queue information to one bit, which distinguishes whether the opponent's queue exceeds a threshold, or not. Each source $i$ starts with one threshold $V_{i}$ (which is greater than $\mu_{i}^{\max }$ ), and transmits with rate $\mu_{i}^{\max }$ if its queue size exceeds $V_{i}$. Source $i$ only needs to know whether the queue backlog $q_{j}(t)$ of the other source $j \neq i$ exceeds the minimum of the maximum service rates of both users $\mu^{\max }=\min _{j=1,2}\left(\mu_{j}^{\max }\right)$, or not. If so, a second threshold $\mu_{\max }$ is introduced. Then, if the queue size of source $i$ is between the two thresholds $\mu_{\max }$ and $V_{i}$, source $i$ transmits with rate $\mu_{\max }$. Shown in Figure 5 , the resulting algorithm is

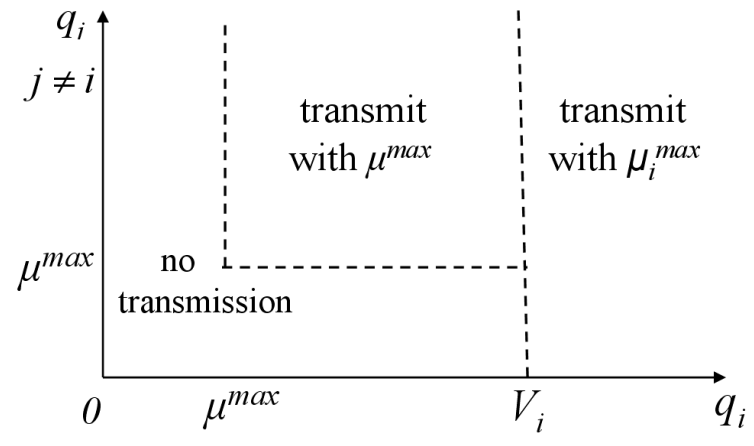

Fig. 5. Algorithm with 1-bit information, (A5), for Source $i . i, j=1,2$.

$\tilde{\mu}_{i}(t)= \begin{cases}0, & \text { if } q_{i}(t)<\mu^{\max } \\ 0, & \text { if } \mu^{\max } \leq q_{i}(t)<V_{i}, q_{j}(t)<\mu^{\max }, j \neq i \\ \mu^{\max }, & \text { if } \mu^{\max } \leq q_{i}(t)<V_{i}, q_{j}(t)>\mu^{\max }, j \neq i, \\ \mu_{i}^{\max }, & \text { if } q_{i}(t) \geq V_{i}\end{cases}$

We will show in Section VIII that the cost performance of (A5) is very close to the centralized algorithm, especially as we increase the threshold $V$. Note that (A5) has a similar structure as the algorithm proposed in [5], where two queues in the relay store packets incoming from two sources. If both relay queues are nonempty, two packets, one from each relay queue, are network-coded and transmitted by the relay. If one of the relay queues is empty, there is no transmission unless the backlogged queue length exceeds a fixed threshold (the buffer capacity). However, in [5], the system is not slotted and the scheduling decisions are carried out at the instants of packet arrivals, and the combined queue state can change at most by 1 , allowing a Markov chain analysis for the decoupled source queues. However, in this paper the number of packet arrivals per time slot may be arbitrary, leading to more complex transitions between the two queue states.

\section{Cost Sharing with Local Queue Information}

Next, we assume that sources do not have any information about each other's queue backlogs and make transmission decisions as in stand-alone operation independent of each other

$$
\tilde{\mu}_{i}(t)= \begin{cases}0, & \text { if } q_{i}(t)=0 \\ u_{i}(t) & \text { otherwise }\end{cases}
$$

The centralized solution $(\mathbf{P 1})$ couples the decisions of the sources through minimizing the cost term $\max \left(\mu_{1}(t), \mu_{2}(t)\right)$, which can be approximated as $\max (x, y) \approx x+y-\left(x^{-\sigma}+\right.$ $\left.y^{-\sigma}\right)^{\frac{-1}{\sigma}}$ for large $\sigma$ such that the centralized solution leads to maximizing

$$
\begin{aligned}
J_{t o t}\left(\tilde{\mu}_{1}(t), \tilde{\mu}_{2}(t)\right)= & \left(q_{1}(t)-V\right) \tilde{\mu}_{1}(t)+\left(q_{2}(t)-V\right) \tilde{\mu}_{2}(t) \\
& -V\left(\tilde{\mu}_{1}(t)^{-\sigma}+\tilde{\mu}_{2}(t)^{-\sigma}\right)^{\frac{-1}{\sigma}}
\end{aligned}
$$

which has the solution of the form:

$$
\tilde{\mu}_{i}(t)= \begin{cases}\min \left(u_{i}(t), f\left(q_{i}(t)\right) \mu_{j}(t)\right), & \text { if } q_{i}(t)<V, \\ u_{i}(t), & \text { if } q_{i}(t) \geq V,\end{cases}
$$

$i \neq j, i=1,2$, where $f\left(q_{i}(t)\right)=\left(\left(1-\frac{q_{i}(t)}{V_{i}}\right)^{\frac{\sigma}{-1-\sigma}}-1\right)^{\frac{1}{\sigma}}$. Each source $i$ has only local queue information and its rate 
TABLE I

Summary of the Proposed Cost Sharing Algorithms

\begin{tabular}{|c|c|}
\hline A1 & Centralized optimal algorithm \\
A2 & Individually optimal algorithm \\
A3 & Worst-case response algorithm \\
A4 & Best-case response algorithm \\
A5 & Dynamic threshold-based algorithm \\
& with 1-bit queue backlog information \\
A6 & Stand-alone algorithm \\
& without queue backlog information \\
A7 & Queue estimation-based algorithm \\
& without queue backlog information \\
\hline
\end{tabular}

linearly depends on the rate of the other source, which is unknown in the decentralized operation at source $i$ and needs to be estimated. Since the rate allocation depends on queue backlog, this involves estimating the queue estimate of the other source. In particular, by assuming that $q_{i}(t)<V$, $i=1,2$, the total cost $J_{\text {tot }}\left(\tilde{\mu}_{1}(t), \tilde{\mu}_{2}(t)\right)$ increases in $\tilde{\mu}_{1}(t)$, if $\min \left(q_{i}(t), \mu_{i}^{\max }\right)<f\left(q_{i}(t)\right) \min \left(\tilde{q}_{j}(t), \mu_{j}^{\max }\right)$, and vice versa. To move the solution to the equilibrium, each source $i$ estimates the queue backlog $\tilde{q}_{j}$ of source $j \neq i$ by solving

$$
\min \left(q_{i}(t), \mu_{i}^{\max }\right)=f\left(q_{i}(t)\right) \min \left(\tilde{q}_{j}(t), \mu_{j}^{\max }\right), i=1,2, i \neq j .
$$

If no valid solution is found for the estimate, source $i$ assumes that queue backlog of source $j$ exceeds $V$ and the other source's rate is $\mu_{j}^{\max }$. After the queue estimates $\hat{q}_{j}(t)$ are found, the rate of the other source $\hat{\mu}_{j}(t)$ is mapped from the queue backlog estimate through an increasing function of $\hat{q}_{j}(t)$ which reaches $\mu_{j}^{\max }$ when $\hat{q}_{j}(t)=V$. In particular, we choose the mapping $\hat{\mu}_{j}=\left(\frac{\hat{q}_{j}}{V}\right) \frac{\mu_{j}^{\max }}{V} \mu_{j}^{\max }$ and each source $i$ independently chooses the rate $\mu_{i}(t)=\min \left(u_{i}(t), f\left(q_{i}(t)\right) \hat{\mu}_{j}(t)\right)$.

\section{Comparison of Cost-Delay Tradeoffs}

We compare the cost and delay of different algorithms summarized in Table I. We assume Poisson traffic with symmetric arrival rates $\lambda_{i}=\lambda$, parameters $V_{i}=V$ and transmission rates $\mu_{i}^{\max }(t)=\mu^{\max }, i=1,2$. The costs are shown in Figures 6 and 7 for $\mu^{\max }=5$ and for $V=10$ and 25 , respectively. The centralized solutions outperform the individually optimal ones. The average costs are high for low arrival rates, since it is less likely that queues are backlogged and network coding opportunities appear. The average cost is reduced, as the parameter $V$ increases.

Figures 8 and 9 depict the average delay as function of the average cost per packet for different values of $V$. For the centralized optimal problem, the usual energy-delay tradeoff is observed, i.e., the energy cost decreases, as delay increases, whereas the individual optimization problems may deviate from this behavior because sources do not have full information on each other's queue backlogs. The average cost per packet achieved by (A5) is very close to the centralized algorithm (A1) and the delay is reduced while achieving the near-optimal cost. This effect is due to (A5)'s property of having a lower threshold, which causes sources to transmit without their queue backlog having to exceed the original threshold $V$ (whenever both source queues are backlogged)

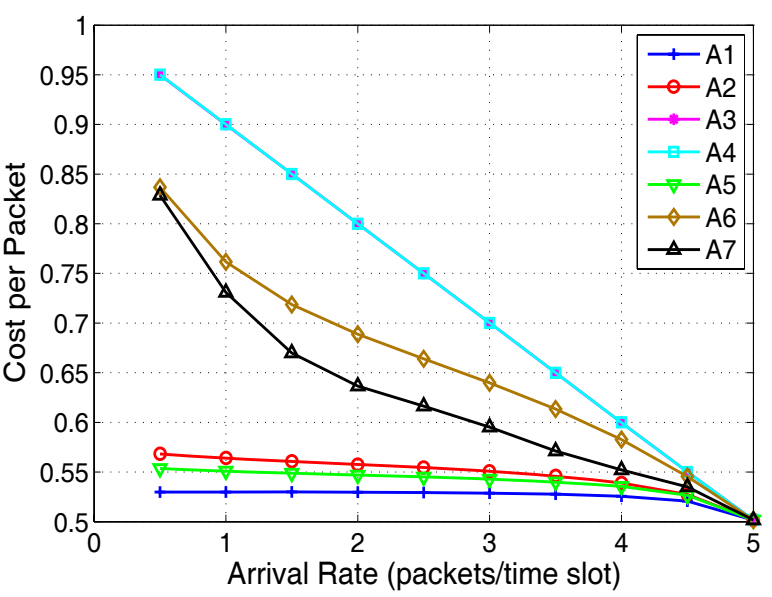

Fig. 6. Cost per packet as function of common arrival rate for $V=10$.

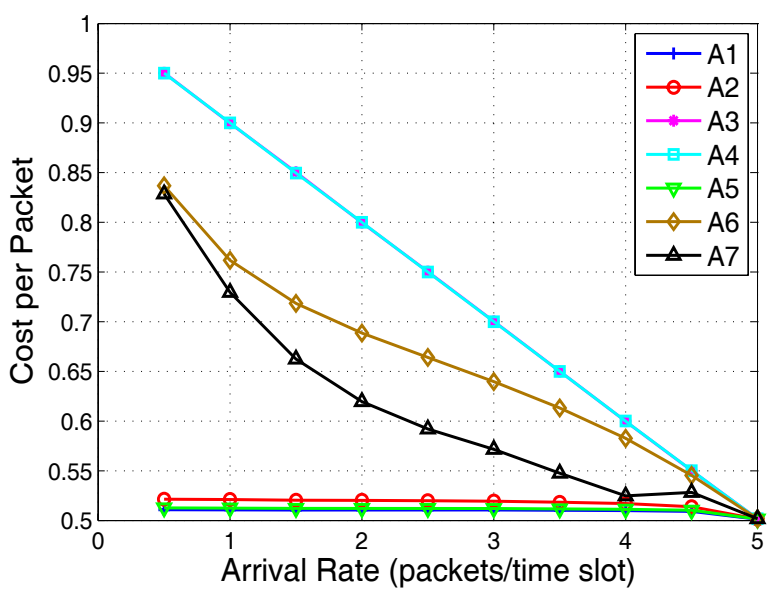

Fig. 7. Cost per packet as function of common arrival rate for $V=25$.

such that the average delay is reduced. It is readily seen that (A7) outperforms other rate allocation algorithms with only local queue information, while providing low delay. Although (A6) provides low delay by always attempting to transmit, the cost per packet might be greater than the optimal value $\frac{1}{2}$ even if both sources transmit simultaneously, since it is likely to have a mismatch between the number of packets transmitted from each source.

\section{CONCLUSION}

We considered the problem of minimizing the cost at a relay node that exchanges packets by network coding or routing depending on the availability of randomly arriving traffic at both sources. The cost is shared by the sources depending on their rate allocation over the relay. We considered different levels of source cooperation and availability of QSI at the sources. First, we derived the centralized control scheme to jointly optimize the cost and stable rates. For distributed operation, sources share the cost of network coding for their packets, whereas the residual cost for plain routing is charged to the source with higher rate requirement.

If sources are selfish with individual objectives, cost sharing with network coding couples them in a rate allocation game. We derived the Nash equilibrium strategies and evaluated the non-cooperative cost-delay tradeoffs. Then, we showed that 


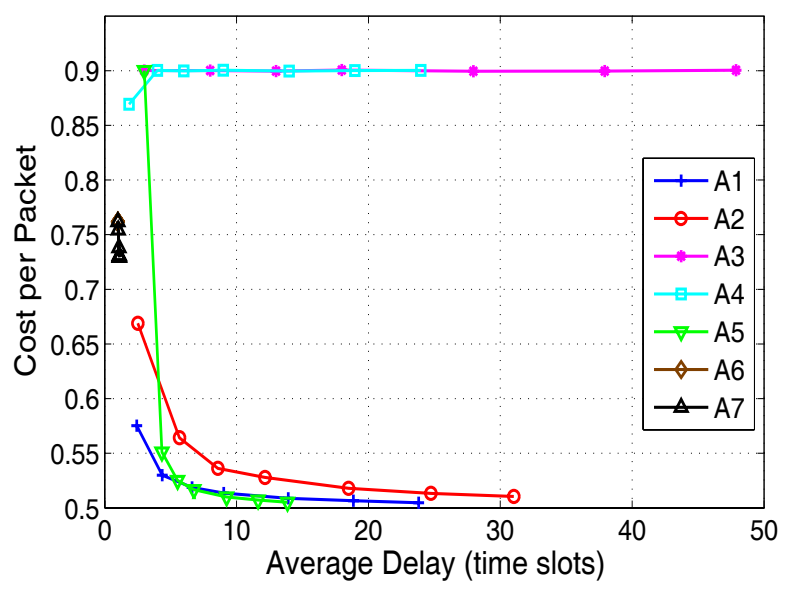

Fig. 8. Cost per packet as function of packet delay, $\lambda=1, \mu_{\max }=5$.

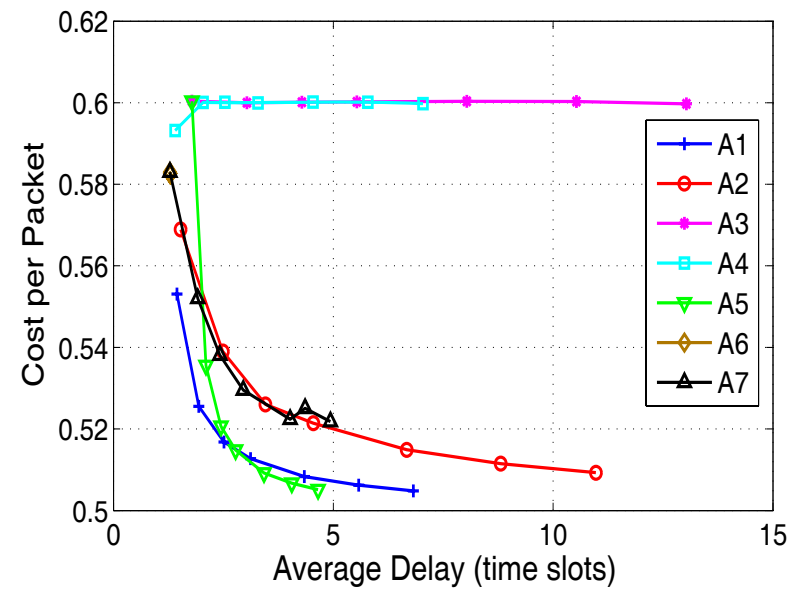

Fig. 9. Cost per packet as function of packet delay, $\lambda=4, \mu_{\max }=5$.

pricing at the relay for the worst-case response can achieve the cost-optimal operation. We also relaxed the QSI assumption and considered simple rate allocation schemes, where sources either have one bit or no information on the queue state of each other. With one bit QSI, the threshold-based algorithms approach the optimal cost, whereas the packet delay does not increase with the threshold as fast as the centralized costoptimal scheme. This leads to new cost-delay tradeoffs for network coding.

Future work includes analysis of an arbitrary number of sources communicating over the relay node. This setting would require understanding multiuser interactions under stochastic traffic that go beyond pairwise packet matching and can lead to interesting new performance tradeoffs.

\section{REFERENCES}

[1] S. Katti, I. Maric, A. Goldsmith, D. Katabi, and M. Medard, "Joint relaying and network coding in wireless networks," in Proc. IEEE International Symp. Inf. Theory, June 2007.

[2] D. S. Lun, N. Ratnakar, M. Medard, R. Koetter, D. R. Karger, T. Ho, E. Ahmed, and F. Zhao, "Minimum-cost multicast over coded packet networks," IEEE Trans. Inf. Theory, vol. 52, no. 6, pp. 2608-2623, June 2006.

[3] Y. Wu, P. A. Chou, and S.-Y. Kung, "Minimum-energy multicast in mobile ad hoc networks using network coding," IEEE Trans. Commun., vol. 53, no. 11, pp. 1906-1918, Nov. 2005.
[4] Y. E. Sagduyu and A. Ephremides, "Cross-layer optimization of MAC and network coding in wireless queueing tandem networks," IEEE Trans. Inf. Theory, vol. 54, no. 2, pp. 554-571, Feb. 2007.

[5] X. He and A. Yener, "On the energy delay trade-off of a two-way relay network," in Proc. Conf. Inf. Sciences Syst., Mar. 2008.

[6] R. Berry and R. G. Gallager, "Communication over fading channels with delay constraints," IEEE Trans. Inf. Theory, vol 48, no. 5, pp. 1135-1149, May 2002.

[7] M. J. Neely, "Optimal energy and delay tradeoffs for multi-user wireless downlinks," IEEE Trans. Inf. Theory, vol. 53, no. 9, Sep. 2007.

[8] M. J. Neely, "Energy optimal control for time varying wireless networks," IEEE Trans. Inf. Theory, vol. 52, no. 7, pp. 2915-2934, July 2006.

[9] E. N. Ciftcioglu, A. Yener, and R. Berry, "Stability of bi-directional cooperative relay networks," in Proc. IEEE Inf. Theory Workshop, May 2008.

[10] E. N. Ciftcioglu, A. Yener, and R. A. Berry, "Stability regions for twoway relaying with network coding," in Proc. Wireless Internet Conf. (invited paper), Nov. 2008.

[11] C. H. Liu and F. Xue, "Network coding for two-way relaying: achievable rate regions, sum rate and opportunistic scheduling," in Proc. IEEE International Conf. Commun., May 2008.

[12] A. Eryilmaz and D. S. Lun, "Control for inter-session network coding," in Proc. NETCOD, Jan. 2007.

[13] T. Ho and H. Viswanathan, "Dynamic algorithms for multicast with intra-session network coding," IEEE Trans. Inf. Theory, vol. 55, no. 2, pp. 797-815, Feb. 2009.

[14] Y. E. Sagduyu, D. Guo, and R. Berry, "Throughput optimal control for relay-assisted wireless broadcast with network coding," in Proc. IEEE International Workshop Wireless Netw. Coding, June 2008.

[15] J. Price and T. Javidi, "Network coding games with unicast flows," IEEE J. Sel. Areas Commun., vol. 26, no. 7, pp. 1302-1316, Sep. 2008.

[16] J. R. Marden and M. Effros, "A game theoretic approach to network coding," in Proc. IEEE Inf. Theory Workshop Netw. Inf. Theory, June 2009.

[17] L. Tassiulas and A. Ephremides, "Stability properties of constrained queuing systems and scheduling properties for maximum throughput in multihop radio networks," IEEE Trans. Automatic Control, vol. 37, no. 12, pp.1936-1948, Dec. 1992.

[18] D. Bertsekas and R. Gallager, Data Networks, 2nd edition. Prentice Hall, 1992.

[19] F. Xue and S. Sandhu, "PHY-layer network coding for broadcast channel with side information," in Proc. Inf. Theory Workshop, Sep. 2007.

[20] N. Bonneau, M. Debbah, R. El-Azouzi, D. S. Menasche, E. Altman, and K. Avratchenkov, "Constrained stochastic games in wireless networks," in Proc. IEEE Globecom, Nov. 2007.

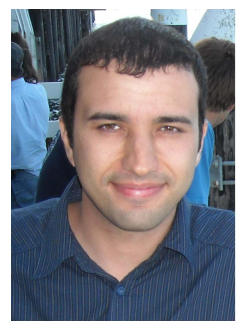

Ertugrul Necdet Ciftcioglu (S'06) received his B.S degree in Electrical and Electronics Engineering from the Middle East Technical University (METU), Ankara, Turkey in 2004 and his M.S. degree in Electronics Engineering and Computer Science from Sabanci University, Istanbul, Turkey in 2006. Currently he is a Ph.D. candidate in the Department of Electrical Engineering, The Pennsylvania State University, PA. He has been a visiting student at Northwestern University, IL in 2009. His research interests are cross-layer design and resource allocation for wireless communication networks, in particular stochastic network optimization for relaying and cooperative communications.

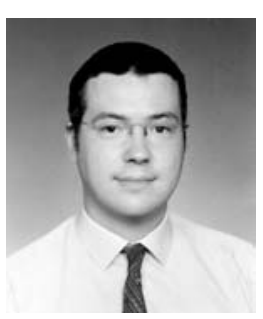

Yalin Evren Sagduyu (S'02-M'08) received the B.S. degree from Bogazici University, Turkey, in electrical and electronics engineering and the M.S. and Ph.D. degrees in electrical and computer engineering from the University of Maryland at College Park. He has been a Graduate Research Assistant with the Institute for Systems Research at the University of Maryland and a Postdoctoral Fellow in the Department of Electrical Engineering and Computer Science at Northwestern University. He is currently a Senior Research Scientist in Intelligent Automation Inc. and a Visiting Assistant Research Scientist in the Institute for Systems Research at the University of Maryland, College Park. His research interests are in the areas of design and optimization of wireless networks, multi-user communications, network coding, information theory, network security and game theory. 


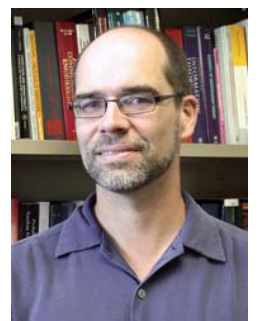

Randall A. Berry (S'93-M'00) received the B.S. degree in Electrical Engineering from the University of Missouri-Rolla in 1993 and the M.S. and PhD degrees in Electrical Engineering and Computer Science from the Massachusetts Institute of Technology in 1996 and 2000, respectively. In 2000, he joined Northwestern University where he is currently a Professor of Electrical Engineering and Computer Science. In 1998 he was on the technical staff at MIT Lincoln Laboratory in the Advanced Networks Group. His primary research interests include wireless communication, data networks, and information theory. Dr. Berry has served as an editor for IEEE TRANSACTIONS ON WIRELESS COMMUNICATIONS and an associate editor for IEEE TRANSACTIONS ON INFORMATION THEORY. He is the recipient of a 2003 NSF CAREER award.

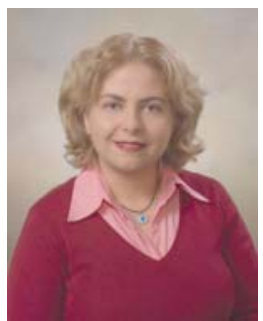

Aylin Yener (S'91-M'00) received two B.Sc. degrees, with honors, in electrical and electronics engineering, and in physics, from Boğaziçi University, Istanbul, Turkey, in 1991, and the M.S. and Ph.D. degrees in electrical and computer engineering from Rutgers University, New Brunswick, NJ, in 1994 and 2000, respectively. During her Ph.D. studies, she was with the Wireless Information Network Laboratory (WINLAB), Department of Electrical and Computer Engineering, Rutgers University. From September 2000 to December 2001, she was a P.C. Rossin Assistant Professor at the Electrical Engineering and Computer Science Department, Lehigh University, PA. In January 2002, she joined the faculty of The Pennsylvania State University, University Park, where she was an Assistant Professor, then Associate Professor, and is currently Professor of Electrical Engineering. During the academic year 2008-2009, she was a Visiting Associate Professor with the Department of Electrical Engineering, Stanford University, CA. Her research interests are in information theory, communication theory and network science, with emphasis on fundamental limits of wireless ad hoc networks and information theoretic security.

Dr. Yener received the NSF CAREER award in 2003 and is a member of the team that received the DARPA Information Theory for Mobile Ad Hoc Networks (ITMANET) Young Investigator Team Award in 2006. Her awards in 2010 include the Penn State Engineering Alumni Society (PSEAS) Outstanding Research Award and the Best Paper award from the Communication Theory Symposium in IEEE International Conference on Communications (ICC). In 2011, she was recognized by the IEEE Information Theory Society for her service as the Student Committee Chair from 2007-2011.

Dr. Yener's service to IEEE includes serving as Technical Program Chair in symposia and tracks in various IEEE conferences including ICC (2008, 2009), PIMRC (2010) and VTC (2012). She served/serves as an editor for the IEEE TRANSACTIONS ON WIRELESS COMMUNICATIONS and for the IEEE TRANSACTIONS ON COMMUNICATIONS. She is the co-founder of the Annual School of Information Theory in North America, an outreach effort of the IEEE Information Theory Society. She served as the general co-chair the first three annual schools of Information Theory in 2008, 2009 and 2010. 